\title{
DESIGN AND DEVELOPMENT OF LIQUID DESICCANT DEHUMIDIFIER FOR AIR CONDITIONING (LDAC) SYSTEM
}

\author{
Digvijay Ramchandra Jadhav \\ Department of Mechanical Engineering \\ Rajarambapu Institute of Technology, \\ Islampur, India
}

\author{
Dr. S. S. Gawade \\ Department of Mechanical Engineering \\ Rajarambapu Institute of Technology \\ Islampur, India
}

\begin{abstract}
In mechanical engineering, air conditioning is emerging area for research. Various of types of liquid desiccants are evolved during the research of LDAC system. Similarly, various kinds of dehumidifier such as falling film, packed bed, bubble and spray dehumidifier are designed. The performance of LDAC system is depend on interaction of liquid desiccants and outside air. The interaction of these fluids depends on density, viscosity, surface tension, flow path, flow direction, viscosity of liquid desiccant, and properties of outside air, out of these properties surface tension, density depends upon types of liquid desiccant. If increase in the velocity of air it will increases in the carrier over loss of liquid desiccant. So, novel flow direction and flow path of liquid desiccant system is developed. Among these various designs suitable design is selected using Pugh Method. Liquid desiccants such as Lithium bromide calcium chloride has been studied. It is obseverd that Calcuim chloride liquid desiccant is suitable as it is easily avalible and has good dehumidification.
\end{abstract}

Keywords - Falling film, Packed bed, Bubble and Spray dehumidifier, Liquid desiccants

\section{INTRODUCTION}

A dehumidifier is an electrical appliance which reduces and maintains the level of humidity in the air, usually for health or comfort reasons, or to eliminate musty odour and to prevent the growth of mildew by extracting water from the air. It can be used for household, commercial, or industrial applications. Large dehumidifiers are used in commercial buildings such as indoor ice rinks and swimming pools, as well as manufacturing plants or storage warehouses. Many indoor sources such as transpiration from occupants, steam from cooking and bath, etc. increase the relative humidity of the air. Nowadays, modern buildings are highly insulated to decrease the loss of energy and which will decrease the infiltration ventilation of air. In this situation, if the inside air is cooled below its dew point temperature it will form dew on windows, doors, walls, etc which can easily turn into moisture causing damage to the woodwork. Dehumidification is important because of some reasons like moulds and fungus attacks, corrosion attack, discomfort due to humid indoor climate, moisture damages on goods, building parts, furniture, etc. When the room is closed very little amount of outside air should be allowed to enter the room. The air in the room is continuously circulated through the dehumidifier and gradually the humidity is condensed into a water container with no resulting heat loss to the outside. When the room is closed there is lower energy consumption, the dehumidification process becomes much faster and easier. Dehumidifiers extract water from air that passes through the unit. There are two sorts of dehumidifiers - condensate dehumidifiers and desiccant dehumidifiers. Condensate dehumidifiers use a refrigerator to collect water known as condensate, which is normally greywater but may at times be reused for industrial purposes. Some manufacturers offer reverse osmosis filters to turn the condensate into potable water. Some designs, such as the ionic membrane dehumidifier, dispose of water as a vapor rather than liquid. Desiccant dehumidifiers (known also as absorption dehumidifiers) bond moisture with hydrophilic materials such as silica gel. Larger commercial units contain hot air recovery systems so as to get rid of humid air from outside the Space. The perfect antidote for a moist, dusty or musty indoor environment is a dehumidifier. The primary function of a dehumidifier is to reduce the amount of humidity in the indoor air but this comes with plenty of secondary benefits such as the removal of dust mites from the atmosphere, reduction in the amount of condensation and an overall improvement in the indoor air quality. A desiccant or compressor dehumidifier will help eliminate some of the common triggers of allergic reactions such as mould, mildew and dust mites. This can be particularly advantageous for people suffering from seasonal allergies. A dehumidifier can also come in handy if used in small or confined spaces with poor ventilation such as in the kitchens and bathrooms. Ideally, these should be built with optimal ventilation that takes out the warm moist air and brings in cool dry air through a convection process. A 


\section{International Journal of Engineering Applied Sciences and Technology, 2020 Vol. 5, Issue 6, ISSN No. 2455-2143, Pages 210-217 \\ Published Online October 2020 in IJEAST (http://www.ijeast.com)}

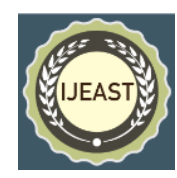

dehumidifier can help to dry laundry indoors quickly, especially during cold winter months.

\section{LITRATURE REVIEW}

This section focuses on the different kinds of experimental design techniques, statistical analysis techniques used for liquid desiccant air conditioning. The LDAC systems are designed since 1980, many components such as dehumidifier, liquid desiccant materials, refrigeration systems are improvised by many authors which are as below: -

W. Z. Gao et al. (2013) [1] have studied that dehumidification effectiveness and moisture removal rate adopted to evaluate and compare with two kinds of dehumidifiers they found that internally cooled dehumidifiers can significantly improve the performance as compared to adiabatic dehumidifiers (cooling water did not flow through the dehumidifier). Lower temperatures of the cooling water can result in higher values for dehumidification effectiveness and moisture removal rate in an internally cooled dehumidifier the limited temperature difference between cooling water and solution might seriously weaken the effect of the cooling water.

K. Sai Kiran et al. (2018) [2] have focused on prototype dehumidifier employing aqueous Lithium Chloride ( $\mathrm{LiCl})$ as desiccant that could accommodate high-influx rates was developed and experiments were conducted to evaluate its performance. Results of the experiments are analysed in terms of performance parameters like moisture removal rate (MRR) and dehumidifier effectiveness. The developed empirical equations were validated by comparing the predicted and measured values on a new set of experiments. They have found that the time-dependent nonlinear response of the system is predicted accurately by the empirical model. The root mean square errors of the observed and predicted values are less than $8 \%$.

Wei Lun et al. (2019) [3] have explained a new method to control the increase in the temperature of a liquid dehumidification solutions presented. A vaporizable coolant (ethanol) was added to a liquid desiccant to form a hybrid solution, and the results indicated that the vaporizing endothermic effect of the vaporizable coolant suppressed the increase in the temperature of the solution. They found that the performance variation in the trends of the conventional $\mathrm{LiCl}$ aqueous solution and self-cooled solution are essentially identical with respect to increases in the parameters of solution or air. Air flow rate and solution temperature negatively affect dehumidification efficiency.

Tao Zhang et al. (2013) [4] have compared the operating performance of an internally-cooled process through both experimental tests and simulation analysis. The predicted results by the established theoretical model agreed well with the experimental results. Based on the validated model, the performance of internally-cooled/heated air processor using liquid desiccant was investigated. They found that low regeneration temperature.

Jason Woods et al. (2018) [5] have focused on internally cooled, 3-fluid exchangers in a parallel plate geometry. Water heats or cools a desiccant across a plate, and the desiccant absorbs or releases water into an airstream through a membrane. They have found that the membrane heat transfer and water heat transfer resistances are the least important. For both the dehumidifier and regenerator, the sensible effectiveness is most sensitive to the air resistance, which accounts for at least $70 \%$ of the overall resistance, and typically more.

Jun Liu et. al (2019) [6] have explained that heat and mass transfer process in internally cooled liquid desiccant dehumidifiers and compared with the typically adopted adiabatic dehumidifier in which three fluids are used inside the dehumidifier. They are air, liquid desiccant, and the internal cooling medium. They found that the enthalpy efficiency of the dehumidifier equals the moisture efficiency $(\eta \mathrm{h}=\eta \mathrm{m})$ when the air inlet state was on the iso-concentration line of the inlet solution.

Anmin $\mathrm{Wu}$ et al. (2006) [7] s that dehumidification performance of liquid desiccant $(\mathrm{LiCl})$ dehumidifier with cooling capacity using compression heat pump system. They have found that the water condensation rate increased with increasing desiccant flow rate, air inlet humidity ratio and desiccant inlet concentration, and changed very little with air inlet temperature and desiccant inlet temperature.

Zili Yang et al. (2016) [8] studied a novel internally-cooled ultrasonic atomization dehumidification system. A model based on the conversion laws of mass and energy, and the sensible heat balance, was developed for performance prediction. Thorough experiments were conducted under various conditions of the airstream, the desiccant solution, and the cooling water to validate the model and good consistency was achieved. The results indicate that dehumidification performance was remarkably improved by $64.9 \%$ in the ICUADS comparing to the adiabatic one. Besides, lower-grade cooling source can be well utilized in the IC-UADS with available cooling-temperature over $25.6 \mathrm{C}$, which could even be from the tap water. Moreover, compared to the packed-bed systems, the ICUADS presented better effectiveness that increased from $47.4 \%$ to $80.1 \%$.

S. Bouzenada et al. (2013) [9] have studied that successive phases of absorption/regeneration, during 7 days by using $\mathrm{LiCl}$ desiccant and on separate phases. The effect of climatic parameters on moisture removal rate and salt concentration on 


\section{International Journal of Engineering Applied Sciences and Technology, 2020 \\ Vol. 5, Issue 6, ISSN No. 2455-2143, Pages 210-217 \\ Published Online October 2020 in IJEAST (http://www.ijeast.com)}

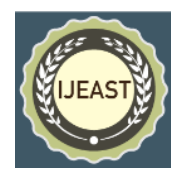

absorption and regeneration processes. They found that higher air humidity gives a higher mass transfer potential then a higher moisture rate absorbed $\mathrm{dm} / \mathrm{dt}$. The decrease of salt concentration affects the $\mathrm{dm} / \mathrm{dt}$ and vapour pressure. Also, these results show that at regeneration temperature, the amount of water desorbed is nearly equal to the amount of water absorbed (equilibrium condition) for a complete cycle. The amount of $7.87 \mathrm{mg}$ of water vapor can be absorbed in the first hour of absorption cycle for $12.6144 \mathrm{mg}$ at $50 \%$ of relative humidity, and $7.004 \mathrm{mg}$ for $36.31 \mathrm{mg}$ of initial mass subjected at $70 \% \mathrm{RH}$. The $\mathrm{LiCl}$ desiccant is able to return to almost its original concentration $31.39 \%$ during regeneration phase. Also, $\mathrm{LiCl}$ desiccant is able to be regenerated at low temperature $40^{\circ} \mathrm{C}$ which can be easily obtained by using solar energy. Then, the $\mathrm{LiCl}$ is a good hygroscopic material for using in liquid desiccant air-conditioning system.

Yilin Liu et al. (2019) [10] have investigated that the performance of cross-flow dehumidifier/regenerator. The empirical correlations of Sherwood number for dehumidification/regeneration were obtained by fitting the experimental data. On the basis of the empirical correlations of Sherwood number and thermodynamics analysis of heat and mass transfer process for dehumidifier/ regenerator, a cross-flow heat and mass transfer model were established. The effects of air and solution parameters on the dehumidification/regeneration performance were analyzed. The number of mass transfer units and the height-to-length ratio of the packing module were also studied. The results show that there exist optimal number of mass transfer units and height-to-length ratio in the dehumidifier/regenerator.

Wei Lun et al. (2018) [11] studied a self-cooled liquid desiccant solution was defined and prepared. Anhydrous ethanol was added into the lithium chloride $(\mathrm{LiCl})$ aqueous solution as the vaporizable coolant to control the increase in the temperature of the solution. A working hypothesis for the self-cooled solution dehumidification was established with a main feature wherein the heat exchangers in conventional internally-cooled dehumidifier were replaced by using selfcooled liquid desiccant solution. Its performance was compared and experimentally investigated. It is observed that the performance indices including moisture removal rate and dehumidification efficiency were analyzed. When compared with conventional $\mathrm{LiCl}$ liquid desiccant solution, the current analysis indicated that the self-cooled solution is superior with respect to the above performance indices.

\section{CONCEPT GENERATION AND SELECTION}

In this section, concept of different types of dehumidifiers are explained. They are explained with their working principles, advantages and disadvantages. The final design of dehumidifier is selected from the following types

\section{A. Three stage Spary type liquid dessicant dehumidifier}

In spray tower dehumidifiers, liquid desiccant is sprayed from the top and the primary air enters from the bottom in counter or cross-flow direction. The desiccant droplets from the top freely descend in the air whilst coming in contact with the rising air stream which allows for heat and moisture transfer between the two fluids. Absence of filling material results in very low air -side pressure drop.

As shown in figure 3.1, conc. Liquid dessicant is sprayed from the top in each stage of dehumidifier. The liquid dessicants are Licl, $\mathrm{CaCl} 2$ or glycols or hygroscopic type. Humidified air or containing moisture is enters the chamber from left side as in figure. The humidified air gets dehumidified partially in the first chamber. Then the partially dehumidified air is transferred to second chamber where it gets dehumidified again. Now also instead of passing air to outside room it is transferred to third chamber for again dehumidification. As the humidified air is passed from three stages moisture absorption will be great. And output air will be more comfortable with less humidity. In each stage the humidified air and liquid dessicant flow is in cross flow direction. After dehumidification, the liquid desiccant solution in each stage becomes diluted. It is collected in at once and transferred to regenerator for removing the exceess water which is absorbed to the atmosphere. The liquid desiccant again becomes concentrated then it will be recirculated to the system.

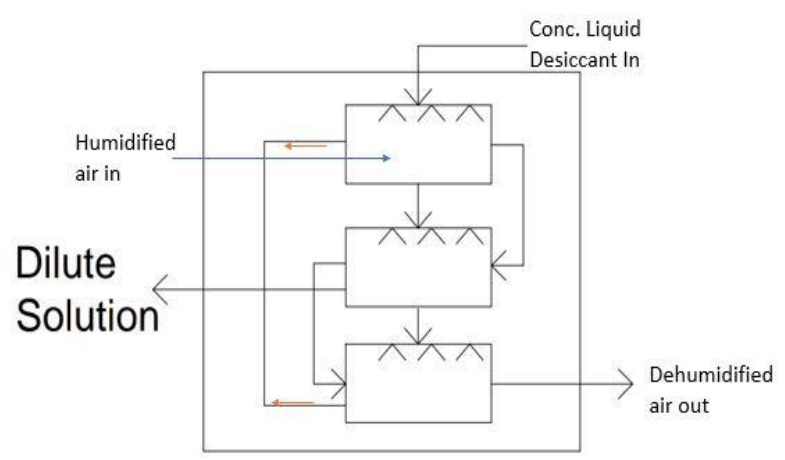

Fig. 3.1:- Three stage Spary type liquid dessicant dehumidifier 


\section{International Journal of Engineering Applied Sciences and Technology, 2020 \\ Vol. 5, Issue 6, ISSN No. 2455-2143, Pages 210-217 \\ Published Online October 2020 in IJEAST (http://www.ijeast.com)}

B. Two stage Solid desiccant and spray type liquid desiccant dehumidifier.

Desiccant dehumidifiers extract moisture from the air. Solid desiccant remove moisture from the air by adsorption. Whenever air dew points approaching zero or lower are desired the refrigerated dehumidifiers are not suitable as cooling air down to these temperatures causes icing on cooling coils thereby affecting the performance of the system. Desiccants such as silica gel or zeolite adsorb moisture at higher temperatures thereby eliminating the coil freeze up issues. The moisture is then removed from the desiccant material by a stream of hot air which is discharged outside the building.

As shown in figure 3.2, conc. Liquid dessicant is sprayed from the top in first stage of dehumidifier. The liquid dessicants are $\mathrm{Licl}, \mathrm{CaCl} 2$ or glycols or hygroscopic type. Humidified air or containing moisture is enters the chamber from left side as in figure. The humidified air gets dehumidified partially in the first chamber. Then the partially dehumidified air is transferred to second chamber where it gets dehumidified again. But in the first chamber there is spray type liquid dessicant dehumidifier and in second stage there is solid desiccant dehumidifier. In second chamber silicon mesh is used as silica gel has moisture removing ability from air. That is in second chamber air gets dehumidified by solid dessicant. In first stage the humidified air and liquid dessicant flow is in cross flow direction. After dehumidification in first stage, the liquid desiccant solution becomes diluted. It is collected and transferred to regenerator for removing the exceess water which is absorbed to the atmosphere. The liquid desiccant again becomes concentrated then it will be recirculated to first chamber.

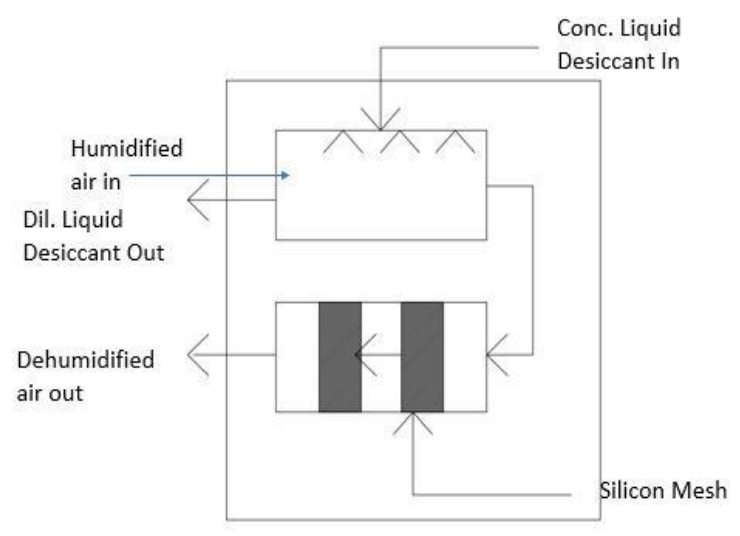

Fig. 3.2 :- Two stage Solid desicaant and spray type liquid desiccant dehumidifier
C. Combination of silicon mesh and falling film dehumidifier

A falling film design comprises of thin plates or tubes over which liquid desiccant is allowed to trickle down under gravity while air is introduced from the top (parallel flow) or bottom (counter flow) or it can be combination of both parallel and counter. The advantages of falling film include low pressure drop on the air side, low desiccant to air flow rates, and lower risk of desiccant carry over.

As shown in figure 3.3, there is single chamber in which both the plates and silicon mesh is included. The plates are mounted like triangle at bottom of chamber and silicon mesh is mounted on the triangle of plate. Humidified air or containing moisture is enters the chamber from left side as in figure. Due to triangle shape inside the chamber it gets directed as shown.

On the triangle of plates conc. liquid desiccant is inserted. It will move on the triangle shape on both sides of plates. On one side of plate it will be in counter flow as liquid and air are in opposite directions. But on other side there will parallel flow of liquid and air. While moving air from one side to another side it will pass through silicon mesh. In silicon mesh, air again gets dehumidified. So there is two times dehumidification in one chamber. Hence it is combination of solid and liquid desiccant dehumidifier in single chamber. After dehumidification, the liquid desiccant solution becomes diluted. It is collected and transferred to regenerator for removing the excess water which is absorbed to the atmosphere. The liquid desiccant again becomes concentrated then it will be recirculated to chamber.

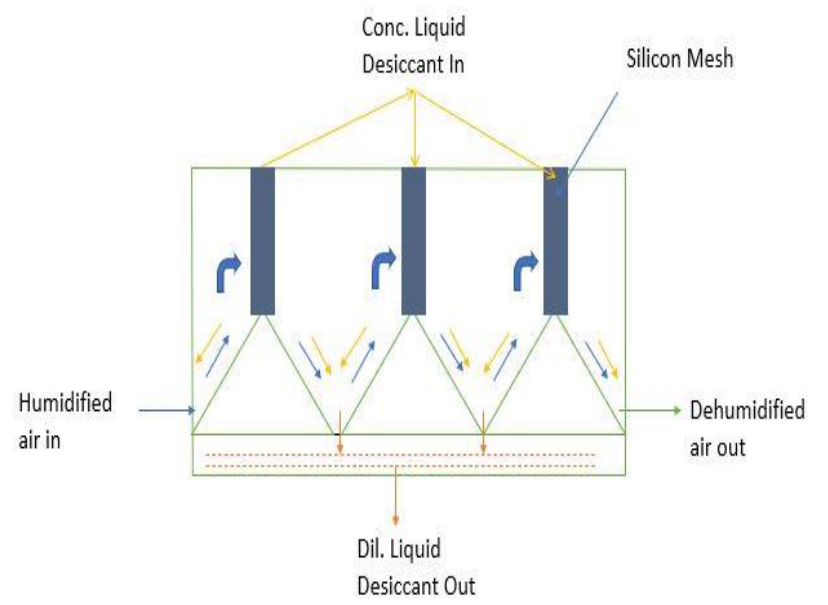

Fig. 3.3: - Combination of silicon mesh and falling film dehumidifier 


\section{International Journal of Engineering Applied Sciences and Technology, 2020 \\ Vol. 5, Issue 6, ISSN No. 2455-2143, Pages 210-217 \\ Published Online October 2020 in IJEAST (http://www.ijeast.com)}

\section{Pipe type liquid desiccant dehumidifier}

Pipe Type liquid desiccant dehumidifier design comprises of tubes into which liquid desiccant is allowed to flow with the humidified air in the same pipe. The Pipe diameter an be larger and flow rate of both liquid desiccant and air is low. This will include low pressure drop on the air side, low desiccant to air flow rates, and lower risk of desiccant carry over.As shown in figure 3.4, there is single chamber in which pipe is used. The pipe is mounted like square 's' shape as in figure. The humidified air and conc. liquid desiccant solution will eneter through the same entry. Due to low flow rate desiccant absorbs moisture from air into pipe. Due to s shape it will act as multistage plate type dehumidifier. After dehumidification, the liquid desiccant solution becomes diluted. It is collected and transferred to regenerator for removing the excess water which is absorbed to the atmosphere. The liquid desiccant again becomes concentrated then it will be recirculated to chamber.

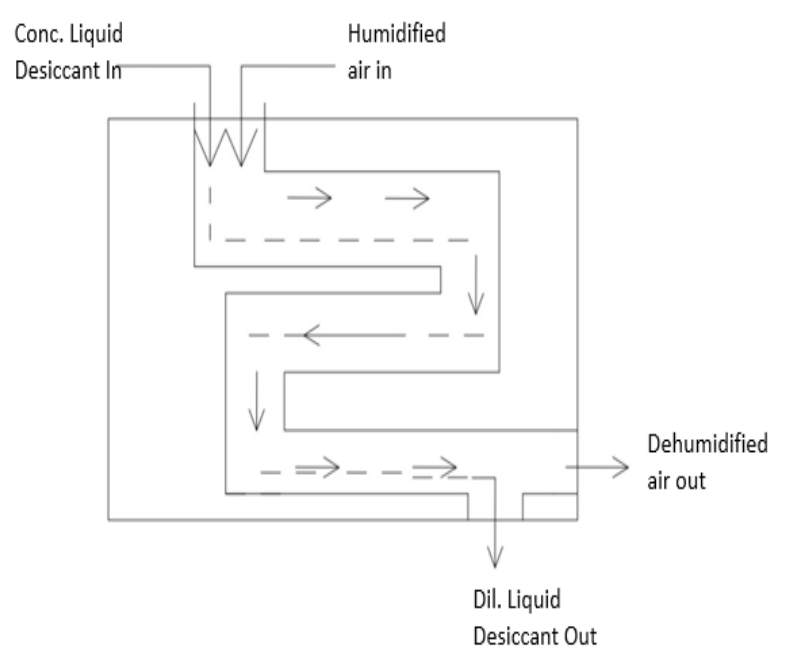

Fig. 3.4:- Pipe Type liquid desiccant dehumidifier

\section{E. Wave Type Structure Dehumidifier}

A falling film design comprises of thin plates or tubes over which liquid desiccant is allowed to trickle down under gravity while air is introduced from one side i.e. cross flow. Liquid desiccant is introduced from the bottom or top of the side. The advantages of falling film include low pressure drop on the air side, low desiccant to air flow rates, and lower risk of desiccant carry over.

As shown in figure 3.5 , there is single chamber in which the plates and for falling film type are included. The plates which are mounted in the chamber are of curved sheet structure. Humidified air or containing moisture is enters the chamber from left side as in figure. Due to curved shape inside the chamber it gets directed as shown. From the bottom of the chamber or top of the chamber of plates conc. liquid desiccant is inserted. It will move on the curved shape on both sides of plates. The interaction between liquid desiccant and humidified air is cross flow type interaction Due to curved shape falling film of liquid desiccant carrier over of solution is in negligible or no carrier over loss of solution. After dehumidification, the liquid desiccant solution becomes diluted. It is collected and transferred to regenerator for removing the excess water which is absorbed to the atmosphere. The liquid desiccant again becomes concentrated then it will be recirculated to chamber. The dehumidified air is taken to room from right side of the chamber.

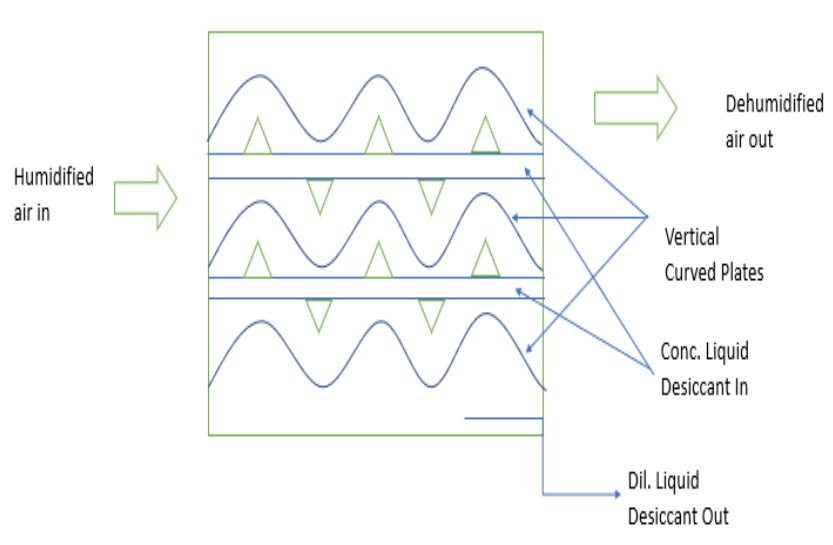

Fig.3.5:-Wave Type Structure dehumidifier

\section{Selection of Best suitable Design}

As discussed in earlier section there are few methods for dehumidification from these best suitable design will be selected for further design and manufacturing. For selection among the various methods pugh method is used.

\section{Pugh Method}

The Pugh Matrix was developed by Stuart Pugh who was a professor and head of the design division at the University of Strathclyde in Glasgow. A well-known inventor in the field of product development and design, management and engineering created a methodical technique that helped identify the most probable solution among all alternatives. This systematic method is called the Pugh Matrix. It is also called a variety of names including Pugh method, Pugh analysis, decision matrix method, decision matrix, decision 


\section{International Journal of Engineering Applied Sciences and Technology, 2020 \\ Vol. 5, Issue 6, ISSN No. 2455-2143, Pages 210-217 \\ Published Online October 2020 in IJEAST (http://www.ijeast.com)}

grid, selection grid, selection matrix, problem matrix, problem selection matrix, problem selection grid, solution matrix, criteria rating form, criteria-based matrix, opportunity analysis. As a decision making model, it is obviously used to choose between a list of alternatives. Now deciding between five alternatives, A, B, C, D and E. We already have a system in place and want to know if one of these five systems would be better for us. Criteria for these selections have been decided. It will be cost, maintaince, ease for manufacturing, modern style, design, size, easy to use, hazardous to envoirment etc. Our baseline is the system we have in place at the moment, so we score this a nought against our criteria.Now consider option A. In relation to criteria 1, we consider that it is better, the same as, or worse than the baseline. If it's better we give it a +1 , if it's the same we give it a 0 , and if it's worse we give it a -1 . Let's say it's +1 . Like this following Pugh matrix is created.

\begin{tabular}{|c|c|c|c|c|c|c|}
\hline Criteria & Baseline/Datum & $\mathrm{A}$ & $\mathrm{B}$ & $\mathrm{C}$ & $\mathrm{D}$ & $\mathrm{E}$ \\
\hline Cost & 0 & + & + & - & 0 & - \\
\hline Maintenance & 0 & + & 0 & 0 & - & 0 \\
\hline $\begin{array}{c}\text { Ease for } \\
\text { manufacturing }\end{array}$ & 0 & 0 & 0 & - & + & 0 \\
\hline Modern style & 0 & 0 & + & + & - & + \\
\hline $\begin{array}{c}\text { Hazardous to } \\
\text { envoirment }\end{array}$ & 0 & - & 0 & + & 0 & + \\
\hline $\begin{array}{c}\text { Size } \\
\text { Easy to use }\end{array}$ & 0 & - & - & + & + & + \\
\hline$\Sigma+$ & & 0 & + & - & + & + \\
\hline$\Sigma-$ & 2 & 3 & 3 & 3 & 4 \\
\hline Toatl & & 2 & 1 & 2 & 2 & 0 \\
\hline
\end{tabular}

Table2.1 :- Pugh Matrix for selection

As Design E has more most probable solution which is more than others amongst five designs. Hence it is selected for manufacturing.

\section{ROPOSED SYSTEM}

1) Working of Liquid desiccant dehumidifier As shown in figure below

1. Air having high humidity enters in dehumidifier chamber from one end exits from another end.

2. In above figure, liquid desiccant flow sprayed over the curved sheets from the bottom of the chamber.

3. The interaction between liquid desiccant and air causes the moisture removal from air due to liquid desiccant properties.
4. Removed moisture with the liquid desiccant is shown by red arrow it will circulated to regenerator to become concentrated so that it can be recirculated to dehumidifier.

5. Dry air is then exits the dehumidifier chamber which is shown by green arrows.

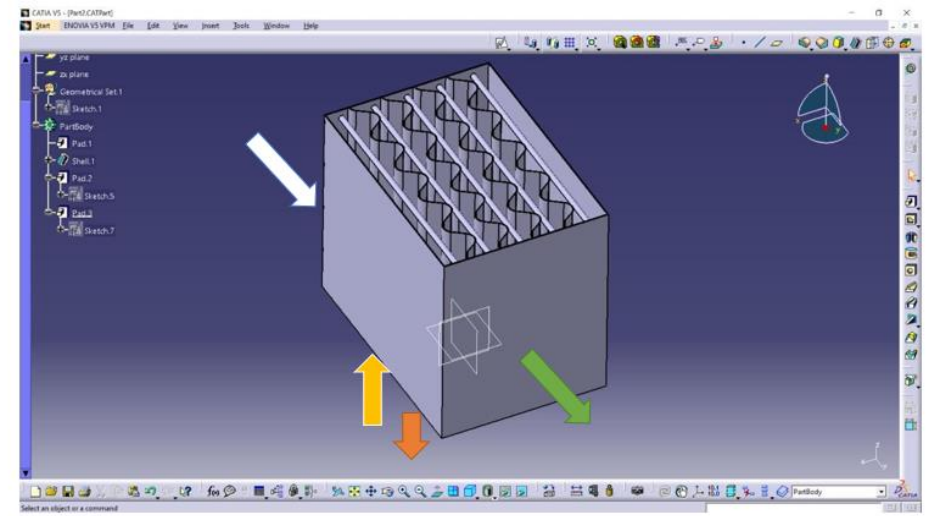

Fig. 4.1:- Working of Liquid desiccant dehumidifier

2) Experimental setup of LDAC system

Liquid desiccant air conditiong system consist of various componets along with dehumidifier. These are important componets for LDAC system. They are fan, pumps, humidifier (required to test the experimental setup) dehumidifier, regenerator, cooling coils or evaporator, psychrometer, liquid desiccant supplying pipes. Schematic of experimental setup of LDAC system is shown below.

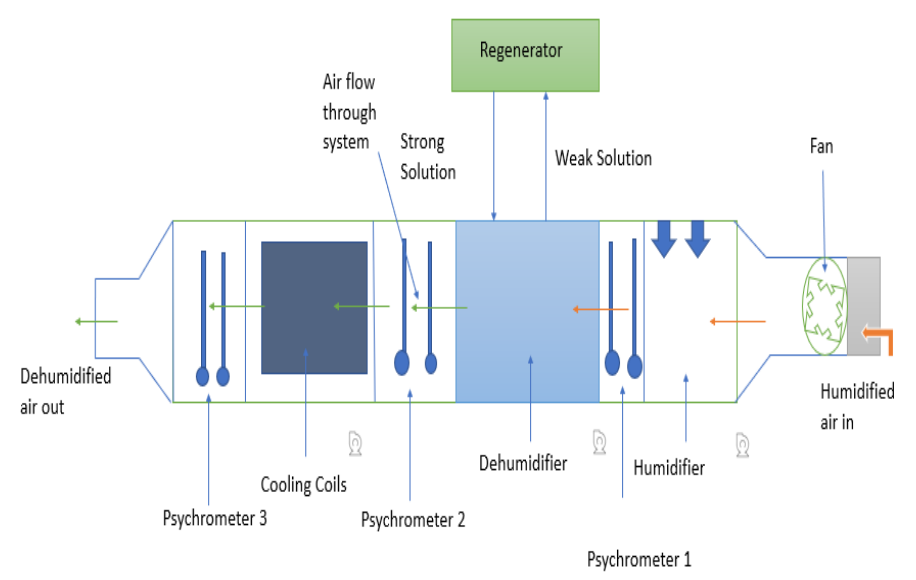

Fig.4.2 :- Schematic of experimental setup of LDAC system

Working of Experimental Setup

As shown in figure above, fan draws the humidified air or wet air from the atmosphere which is shown in the figure by orange arrows to the right-hand side. Then the air is passed to the humidifier which is spray type humidifier here moisture 


\section{International Journal of Engineering Applied Sciences and Technology, 2020 \\ Vol. 5, Issue 6, ISSN No. 2455-2143, Pages 210-217 \\ Published Online October 2020 in IJEAST (http://www.ijeast.com)}

can be added to the air for dehumidification. Psychrometers are used for sensing the dry bulb temp. and wet bulb temp. After that the air is passed to dehumidifier where moisture is removed from the air. The desiccant solution which became weak is transferred to the regenerator for making it concentrated again. Strong solution again circulated in dehumidifier for further use. After dehumidification psychrometer 2 measures the dry bulb temp. and wet bulb temp from that we can get the relative humidity. Now the air is passed on cooling coils or evaporator so that temp. of air can be reduced and air will get cool. Again psychrometer 3 measures the DBT and WBT. The dehumidified cool air is then passed to the living room.

\section{3) Calculations}

Initial conditions used during experimentation-

Atmospheric temperature $=30^{\circ} \mathrm{C}$

Relative humidity $=75$ to $80 \%$

Absolute humidity $=21.1 \mathrm{~g} / \mathrm{kg}$

Air density $=1.22 \mathrm{~kg} / \mathrm{m}^{3}$

Amount of water vapour in $1 \mathrm{~m}^{3}$ of air

$=($ Air density $\times$ Humidity $) / 1000$

$=(1.22 \times 21.1) / 1000$

$=0.0257$

Air flow Rate $=\mathrm{CFM}($ Cubic feet per minute $)$

Length $\mathrm{x}$ Width $\mathrm{x}$ Height $\mathrm{x}$ (Air Changes per Hour)

$=\longdiv { 6 0 \operatorname { m i n } } \begin{array} { c } { \frac { 1 0 \times 1 0 \times 1 2 \times 6 } { 6 0 \mathrm { min } } } \\ { = 1 2 0 \mathrm { cfm } } \end{array}$

Air flow rate $=3 \mathrm{~m}^{3} / \mathrm{min}$

Amount of water vapour per $\min$ in air $=0.025742 \times 3$

$$
=0.0772 \mathrm{~kg} / \mathrm{min}
$$

Solution absorbing capacity $=0.8 \mathrm{~kg} / \mathrm{kg}$

Amount of solution req

$$
\begin{aligned}
& \text { Amount of water vapor per min in air } \\
& \hline \text { Absorbing Capacity of solution } \\
&=0.077226 / 0.8 \\
&=0.09653 \mathrm{~kg} / \mathrm{min} \\
&=0.000077226 \mathrm{~m}^{3} / \mathrm{min} \\
&=0.077226 \mathrm{lpm}
\end{aligned}
$$

Factor of safety $=2$

Amount of Solution after considering FoS

$=0.154452 \mathrm{lpm}$

Maximum running time required $=10$ hours

Total Solution required $=$ Amount of Solution $\times$ Maximum timex $60=92.671$ Litres

\section{CONCLUSION}

Hence from the above, the falling film, packed bed, bubble and spray dehumidifiers has been studied. Different types of concepts of dehumidifier has been developed. By using the Pugh Method best suitable model is selected for further development. Selected model is of falling film type dehumidifier which is good for better dehumidification of air. The design has novel flow direction of air and liquid desiccant i.e. commination of parallel and counter flow. In order to test the dehumidifier, humidifier is also used here to humidify air and achieve $65-70 \%$ humidity. Human comfort humidity is $40 \%-60 \%$. Flow rate of air in room of size $10 \mathrm{~m} \times 10 \times 12 \mathrm{~m}$ is found to be $120 \mathrm{cfm}$ i.e. $0.0567 \mathrm{~m}^{3} / \mathrm{s}$.

\section{REFERENCES}

[1] Gao W.Z., Shia Y.R., Chengb Y.P., Suna W.Z., (2013), "Experimental study on partially internally cooled dehumidification in liquid desiccant air conditioning system”, Building and Environment 63 (pg202-209)

[2] K. Sai Kiran, Benny Raphael, (2018), "Performance Evaluation of a High-Influx, Bubble dehumidifier"

[3] Liu Jun, Liu Xiaohua , Zhang Tao, (2019), “Analytical solution of heat and mass transfer process in internally cooled liquid desiccant dehumidifiers using refrigerant as cooling medium", Energy \& Buildings 190, (page 1-14)

[4] Zhang Tao, Liu Xiaohua, Jiang Jingjing, Chang Xiaoming, Jiang Yi, (2013), "Experimental analysis of an internally-cooled liquid desiccant dehumidifier", Building and Environment 63, (pg1-10)

[5] Woods Jason , Kozubal Eric, (2018), “On the importance of the heat and mass transfer resistances in internallycooled liquid desiccant dehumidifiers and regenerators", International Journal of Heat and Mass Transfer 122, (pg324-340)

[6] Liu Jun, Liu Xiaohua, Zhang Tao, (2019) ,“Analytical solution of heat and mass transfer process in internally cooled liquid desiccant dehumidifiers using refrigerant as cooling medium”, Energy \& Buildings 190, (pg1-14)

[7] Anmin Wu, Chunlin Li, Hefei Zhang, (2006) "The Primary Research on Liquid Desiccant Dehumidifier With Cooling Capacity Using Compression Heat Pump System", International Refrigeration and Air Conditioning Conference at Purdue, (pg17-20),

[8] Yang Zili, Tao Ruiyang, Hui Ni, Zhong Ke , Lian Zhiwei, (2016) ,"Performance study of the internallycooled ultrasonic atomization liquid desiccant dehumidification system", Building and Environment 103 134-145

[9] Bouzenadal S., Kaabi A. N., Fraikin L. and Léonard A., (2013), "Successive Dehumidification/Regeneration Cycles by LiCL Desiccant for Airconditioning System", Journal of Renewable and Sustainable Energy 5, 023134

[10] Liu Yilin, Jin Liwen, Yang Jin, Yang Xiaohu, Meng Xiangzhao \& Lianying, (2019) "Experimental and 
Numerical Study on Heat and Mass Transfer of Cross-

Flow Liquid Desiccant Dehumidifier/Regenerator", Heat Transfer Engineering

[11] Lun Wei, Li Kuining, Liu Bin, Zhang Hongji, Yang Yuxiao, Yang Cheng, (2018) "Experimental analysis of a novel internally-cooled dehumidifier with self-cooled liquid desiccant", Building and Environment 141 (pg117126) 